\title{
FastSME: faster and smoother manifold extraction from 3D stack
}

Basu, Sreetama; Rexhepaj, Elton; Spassky, Nathalie; Genovesio, Auguste; Paulsen, Rasmus Reinhold; Shihavuddin, ASM

Published in:

Proceedings of the IEEE 2018 Conference on Computer Vision and Pattern Recognition

Publication date:

2018

Document Version

Publisher's PDF, also known as Version of record

Link back to DTU Orbit

Citation (APA):

Basu, S., Rexhepaj, E., Spassky, N., Genovesio, A., Paulsen, R. R., \& Shihavuddin, ASM. (2018). FastSME: faster and smoother manifold extraction from 3D stack. In Proceedings of the IEEE 2018 Conference on Computer Vision and Pattern Recognition IEEE.

\section{General rights}

Copyright and moral rights for the publications made accessible in the public portal are retained by the authors and/or other copyright owners and it is a condition of accessing publications that users recognise and abide by the legal requirements associated with these rights.

- Users may download and print one copy of any publication from the public portal for the purpose of private study or research.

- You may not further distribute the material or use it for any profit-making activity or commercial gain

- You may freely distribute the URL identifying the publication in the public portal 


\title{
FastSME: faster and smoother manifold extraction from 3D stack
}

\author{
Sreetama Basu ${ }^{1}$, Elton Rexhepaj ${ }^{2}$, Nathalie Spassky ${ }^{1}$, Auguste Genovesio ${ }^{1}$, Rasmus Reinhold \\ Paulsen $^{3}$ and ASM Shihavuddin ${ }^{3}$ \\ ${ }^{1}$ Department of Biologie, Ecole Normale Superieure, Paris, France., sreetama.basu@ens.fr, \\ spassky@biologie.ens.fr, auguste.genovesio@ens.fr \\ ${ }^{2}$ Institut Curie, Paris, France. elton.rexhepaj@curie.fr \\ ${ }^{3}$ Technical University of Denmark, Copenhagen, Denmark.rapa@dtu.dk, shihav@dtu.dk
}

\begin{abstract}
$3 D$ image stacks are routinely acquired to capture data that lie on undulating $3 D$ manifolds yet processed in $2 D$ by biologists. Algorithms to reconstruct the specimen morphology into a $2 D$ representation from the $3 D$ image volume are employed in such scenarios. In this paper, we present FastSME, which offers several improvements on the baseline SME algorithm which enables accurate $2 D$ representation of data on a manifold from $3 D$ volumes, however is computationally expensive. The improvements are achieved in terms of processing speed (3X-10X speed-up depending on image size), minimizing sensitivity to initialization, and also increases local smoothness of the recovered manifold resulting in better reconstructed $2 D$ composite image. We compare the proposed FastSME against the baseline SME as well as other accessible state-of-theart tools on synthetic and real microscopy data. Our evaluation on multiple metrics demonstrates the efficiency of the presented method in maintaining fidelity of manifold shape and hence specimen morphology.
\end{abstract}

\section{Introduction}

Microscopy images have become one of the primary ways to explore, analyze and quantify various biological phenomena. The rapid progress of automated microscopy technology in the last decade enables the acquisition of biological samples as images with varying scale and resolutions. Low-resolution high through-put images are used in pharmacology and basic biology to generate and prioritize hypotheses which is further investigated and validated via high resolution widefield and confocal microscopy images in labs. Biological specimens, having thickness and surface profiles considerably larger than the focus range of microscope lenses, are frequently imaged as $3 \mathrm{D}$ volumes. Optical sectioning of the specimen captures the in-focus information at increasing depths simulating an extended depth of field. A 3D image stack is thus a sequence of focused image slices, which can subsequently be processed to reconstruct a single all-datain-view image. In bio-image analysis, 2D projection of volume images are routinely used for dimensionality reduction to visualize and analyze various data [15]. The objective for a $2 \mathrm{D}$ approximation of the $3 \mathrm{D}$ volume is to maximize information content maintaining accuracy of manifold shape and simultaneously minimizing the distortion of Point Spread Function (PSF) noise and imaging artifacts.

In this paper, we use the term composite image for the recovered 2D representation of the 3D image stack data and index map for the Z-values on the manifold to retain depth information. Due to limited depth of field of microscope lenses' focus, these methods were initially conceived for bright field light microscopy [1, 14]. The growing popularity of 3D confocal microscopy has generated new demands on traditional composite image generation algorithms. Extraction of the manifold in a 3D microscopy image volume containing the specimen of interest begins with acquisition of an extended depth of field image stack. We apply an operator, for example intensity maxima, in case of Maximum Intensity Projection (MIP) on each 2D image slice. The plane index of maxima of intensity response for each pixel position along $\mathrm{z}$-axis is selected as the decision map from which to create the final composite image. In the literature, several methods have been studied for specific applications and imaging modalities $[17,11,5,3]$. Most of these fails when presented with very diverse specimen morphologies as they have been over-optimized for specific applications. On the other 
hand, methods that can address diverse imaging modalities and specimen morphology, fail to create local coherence of the extracted manifold. It is mainly because 3D volume images of biological specimen present a distinct foreground and background. The foreground is the deliberately labeled high intensity pixel that move in and out of field of view on a plane. However, it is tricky to determine when the unlabeled background is following the manifold in consistence with the foreground. These regions often present no intensity variation or texture. As a result, in case of pixel wise projection methods, composite images are often assigned values from the maximally noisy planes. Hence, existing methods tend to pick up the background in the index map from defocused planes in the $3 \mathrm{D}$ volume accumulating PSF noise and background artifacts and create a false distortion of the specimen morphology. In our framework, we give strong emphasis on spatial coherence of the manifold's depth index to extract a better representation of the data. These methods have the potential to be useful in bio-image analysis from 3D microscopy data to obtain a high-quality, meaningful and denoised image of reduced dimensionality with relevant adaptations.

\section{Previous works}

ImageJ [13] and Cell Profiler [7] are the freely available microscopic image analysis softwares that are most frequently used by biologists. These tools offer several primitive focus operators like Maximum/minimum intensity projection (MIP), standard deviation in z-axis, average intensity projection etc. However, there is no control on the smoothness or shape of the recovered manifold, giving the biologists no opportunity to ensure local neighborhood coherence of the reconstructed 2D composite image, as expected in natural biological specimen. Another ImageJ Plugin, Extended Depth of Field (EDF)[1] gives the user control over smoothness of the 3D map, however it was conceived for grayscale images. In case of color images, it forces the $2 \mathrm{D}$ approximation to take place in parallel, independently on each of the color channels. In biological studies, it is often the case that one channel works as a reference from which to infer the orientation of the 2D manifold in 3D space and then to apply the same index map to all channels. For example, in co-localization studies of proteins along a cell membrane layer with cell-membrane and protein of interest imaged in separate channels. A recent review article on ContentAware Image Restoration [19] highlights this problem and the many recent algorithmic toolkits that try to address it $[8,4,15]$. Another relevant recent method is Premosa [4] - a multi scale analysis toolkit to quantify how cellular processes create tissue dynamics. This method works very accurately even on very noisy data and can be very efficient in time. However the method is not suitable to use in widefield microscopy and tends to over smooth the planes with high curvature as demonstrated in the results section 4 . The SME method [15] produced the most accurate $2 \mathrm{D}$ projection for both confocal and widefield imaging modality, however the method is computationally expensive and requires many iterations to position the background manifold in coherence with the local foreground depths. The SME method have been already been used for various biological studies as the $2 \mathrm{D}$ projection algorithm in works described in the [9, 2, 16].

In FastSME, we address the above mentioned limitations of the SME method and propose modifications than can produce faster, smoother and more accurate $2 \mathrm{D}$ reconstruction of the 3D image stack. FastSME is applicable as a preprocessing step in many modalities of microscopy imaging where data lies on a manifold in 3D volume but requires a $2 \mathrm{D}$ representation for visualization and analysis. We demonstrate several applications and made a MATLAB implementation available for similar tasks. In this method, the focus in on the analysis of 2D manifolds in a $3 \mathrm{D}$ space frequently referred to as $2.5 \mathrm{D}$ imaging in the literature. However, it is critically important to determine if a $2 \mathrm{D}$ projection image creates a distortion of the actual morphology. While recovering the shape of a 2D manifold such as the cell membrane, projection is an appropriate operation. In contrast, a 3D cell imaged as a 3D volume, will suffer significant distortion of morphology with any of the dimensionality reduction methods through projection methods and hence is better analyzed in 3D. Hence, given the impossibility of exhaustively listing all applicable scenarios, ultimately, domain experts' discretion is advised in the use of any projection to 2D from 3D image volume for visualizing or quantifying a biological specimen.

\section{Contributions}

The main contributions of this paper is an improved version of the SME algorithm [15]. The improvements are two-fold.

1. Significant reduction in computational cost: FastSME deploys a gradient descent momentum optimization scheme on a novel per-class cost function with a multi-scale approach making it useful to be applied in practice to larger image data - the speedup observed is $3 \mathrm{X}-10 \mathrm{X}$ relative to the size of the image. This is enforced through the adoption of a pyramidal scheme multi-scale optimization of the index map and adoption of stochastic gradient descent momentum [12] with simulated annealing of the step size for the novel energy functional minimization.

2. Efficient propagation of foreground manifold to 
background: Secondly, the qualitative improvement of the obtained index map (which in turn results in an improved reconstructed composite image, although it is at times visually imperceptible or by adopted evaluation metrics). Information propagation regarding depths of foreground pixel to the background pixel locations on the index map is impeded in the iterative optimization process with gradient decent as the energy function can get trapped in local minima within few iterations. The improved per-class cost function and optimization strategy implies FastSME can start to place the background index map in close proximity to the foreground much faster in the optimization run before the step size becomes very small allowing significantly higher local consistency of index map to be achieved.

The rest of the article is organized as follows: In section 4 we present our proposed algorithm with specific attention to the improvements in FastSME over SME. Section 5 introduces the evaluation metrics and synthetic data we used in order to compare performance of FastSME and state-of-the-art methods. We further demonstrate the performance of FastSME on several diverse image data set acquired by different imaging modalities and containing diverse specimen morphologies. Finally we present the discussion of the experimental results and conclude in section 6 .

\section{Proposed Method}

Our primary objective is to estimate the manifold passing through the foreground signal and propagate it smoothly through the background regions. SME formulates equation 1 in a cost minimization framework where the overall cost is the sum of the distance from the index map $Z$ to the most focused $\mathrm{z}$ level, $Z_{\max }$ and the local variance of the index map, $\sigma_{z}$. Firstly, we need to determine if pixels belong to foreground or background based on their $\mathrm{z}$ profile. This is performed using 3 class kmeans [6] on the frequency domain representation of the $z$ profiles and classes are assigned based on high power, low frequency profiles to foreground, medium-frequency profiles to an uncertain class and high frequency profiles to background class. The per class weighting factor $C$ between $\mathrm{z}$ error term and regularization term is estimated from the data as described in details in [15]

$$
Z^{*}=\operatorname{argmin} \sum_{(x, y)}\left[C\left|Z_{\max }-Z\right|+\sigma_{z}\right]
$$

\subsection{Normalized cost function}

In the FastSME method, the cost function is designed to find the optimal index map $Z$ (used to reconstruct a

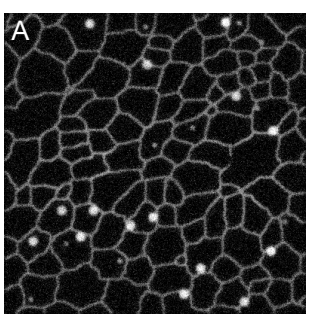

SYNTHETIC TISSUE

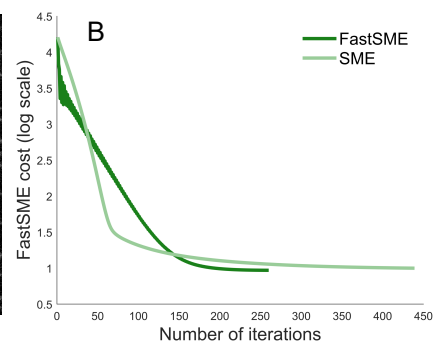

Figure 1. Figure demonstrates the improvement in optimization of cost function (B) achieved by FastSME (dark green) compared to SME (light green) on 'SYNTHETIC TISSUE' dataset (A).

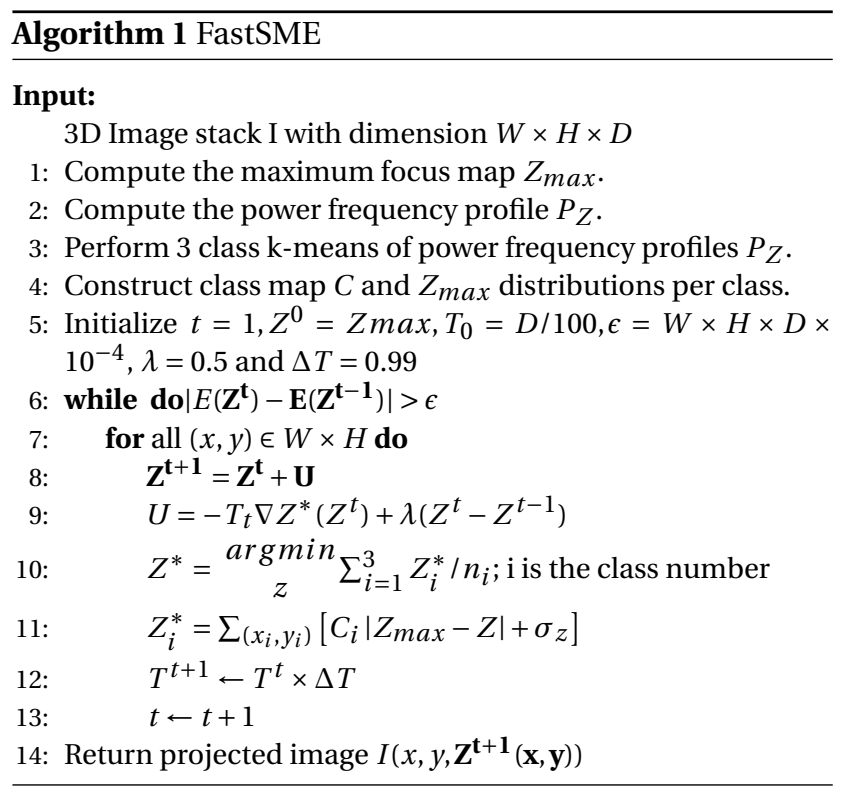

2D composite image from the 3D stack), that minimizes the distance to the maximum focus map $Z_{\max }$ (ensuring its foreground proximity) and local variance of $Z$ (ensuring its smoothness in $3 \times 3$ neighborhood). The proposed modification to the cost function is not to normalize the cumulative contribution of all the three classes derived from k-means of the frequency domain representation of the $\mathrm{z}$ profiles (background, uncertain and foreground class) by the total number of pixels. This tends to undeservedly prioritize is it the smoothness in case of background class and the depth levels in case of foreground class (which ever is the largest by pixel number class in the classmap referring Figure 2 B) in the final derived index map. In FastSME, we normalize the cost contribution of each class by their respective cardinality [equation 2], thus modifying the overall cost to the sum of normalized cost per class [equation 3]. This modification enables the recovered index map to be less sensitive to its initialization. Currently the index map is still initialized from Maximum Intensity Projection (MIP) similar to SME 


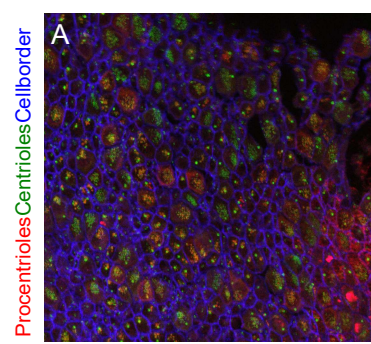

EPENDYMAL CELLS

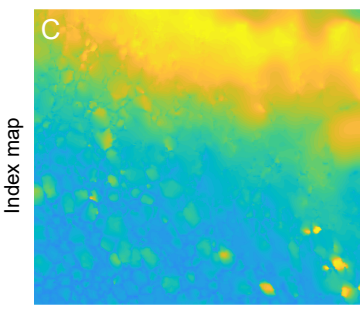

FastSME

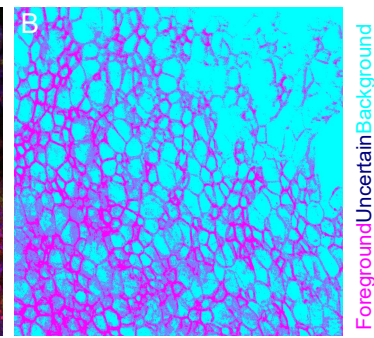

Classmap

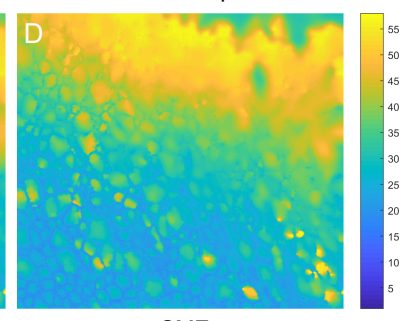

SME
Figure 2. Figure demonstrates the improvement in quality of recovered index map in terms of local coherence while preserving the original biological structure (which in this case is apical layer tissue surface) achieved by FastSME (C) compared to baseline SME (D) on 'EPENDYMAL CELL' dataset (A) using classmap in $\mathrm{B}$. The scalebar indicates the stack depth of the $3 \mathrm{D}$ volume 1 (dark blue) indicates the top of the stack and 58 (yellow) indicates the bottom stack.

but in practice can be initialized randomly. Here $Z^{*}$ is the final index map which is derived from the optimization scheme by minimizing the summation of cost $Z_{i}^{*}$ per class denoted by class identity $i$ (including foreground $c_{f}$, uncertain $c_{u}$ and background class $c_{b}$ ). $n_{i}$ is the number of pixels belonging to class $i$. $\sigma_{z}$ is the regularization term and $C$ is the respective class weight as described more in details in [15]

$$
\begin{gathered}
Z_{i}^{*}=\sum_{\left(x_{i}, y_{i}\right)}\left[C_{i}\left|Z_{\max }-Z\right|+\sigma_{z}\right] \\
Z^{*}=\operatorname{argmin} \sum_{i=1}^{3} Z_{i}^{*} / n_{i}
\end{gathered}
$$

\subsection{Gradient descent with momentum optimization}

In FastSME, the optimization scheme employed is âĂŸstochastic gradient descent with momentumâĂŹ [12] . Gradient descent scheme in general updates weights and biases to minimize the loss function by taking steps in the direction of the negative gradient of the loss.

$$
Z^{t+1}(x, y)=Z^{t}(x, y)-T_{t} \nabla Z^{*}\left(Z^{t}(x, y)\right)
$$

where $t$ stands for the iteration number, $T_{t}>0$ is the step size, $Z^{t}(x, y)$ is the individual $Z$ position of the index map at $t$ th iteration, and $\nabla Z^{*}\left(Z^{t}(x, y)\right)$ is the gradient of loss function at $Z^{t}(x, y)$.

For the 'stochastic gradient descent with momentum' scheme the momentum term is added to regularize the index map adjustment process [Equation 5]. Addition of the momentum term to the index map optimization scheme steadies the rate of cost drop and makes a faster descent towards the global minima. It also helps to avoid getting stuck in saddle points in the solution space.

$$
\begin{gathered}
U=-T_{t} \nabla Z^{*}\left(Z^{t}(x, y)\right)+\lambda\left(Z^{t}(x, y)-Z^{t-1}(x, y)\right) \\
Z^{t+1}(x, y)=Z^{t}(x, y)+U
\end{gathered}
$$

$\lambda$ is the momentum weight that defines the contribution of the previous gradient step to the current step. $U$ is the final update factor to the index map $Z^{t}(x, y)$. The step size is initialized to $T_{t}=D / 100$, where $D$ is the number of planes in the image stack. Multiplying it by $\Delta T$ at each iteration, produces a geometric decay scheme that ensures faster convergence and reduces possibility of oscillation during optimization. Empirically, in all datasets $\Delta T=0.99$ and momentum weight $\lambda=0.5$ gave satisfactory results. Momentum weight of 0.5 implies that the update factor $U$ contains the same contribution from the current gradient and the current velocity. Other available state-of-the-art optimizers such as adam [10], adadelta [20], rmsprop [18] when tested, produced almost similar result with varying number of iterations. 'stochastic gradient descent with momentum' produced consistently lower cost with fewer iterations on different initializations for the index map for the 'SYNTHETIC TISSUE' dataset and hence we choose this optimizer. The stopping criteria is being set as $\nabla Z^{*} \leq W \times H \times D \times 10^{-5}$ instead of $\nabla Z^{*} \leq W \times H \times D \times 10^{-6}$; where $W, H, D$ are the width, height and depth of the input 3D stack respectively. This relaxation of the stopping criteria reduces required number of iterations. In gradient descent optimization in the pilot SME, the later iterations affected only floating point adjustments on the index map that does not impact the quality of the composite image significantly because it is only possible to use integer values when generating the composite image from the index map as we do not interpolate intensity values between 2 consecutive $Z$ planes.

Figure 1 illustrates the iterative optimization process of SME and FastSME runs of the 'SYNTHETIC TISSUE' dataset (in plot A). In FastSME, the cost reached 0.9708 (in log scale) with 260 iterations much faster than the cost minima reached by SME which is 1 (in log scale) with 439 iterations. In SME optimization as illustrated in Figure 1 $\mathrm{B}$ in light green, the cost function has a rapid decrease in the initial iterations because it smooths the index map 


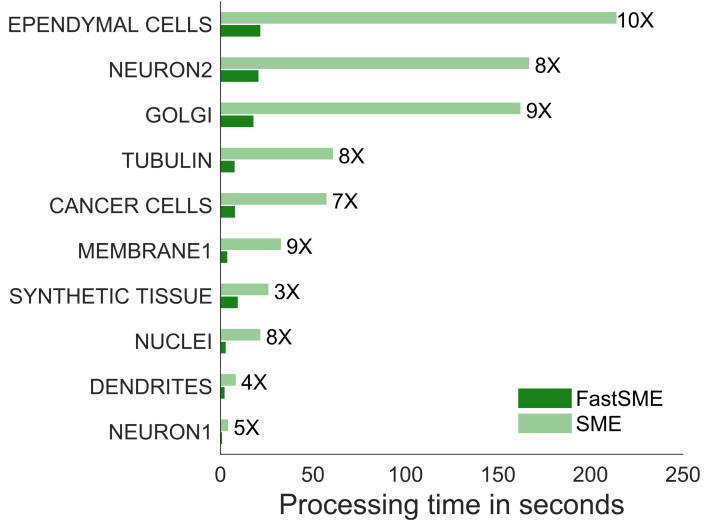

Figure 3. FastSME achieve on average 7X speed gain on SME. On various biological datasets the obtained gain is in range of $3 X$ to $10 X$. The speed gain is directly proportional to total number of pixels on the index map. Datasets are ordered in sequence of increasing $2 \mathrm{D}$ image sizes.

from initial maximum focus map which is locally incoherent. In later parts of the run, the step size gets smaller, therefore it takes many more iterations to propagate the foreground manifold to the background manifold hence minimizing the cost $\nabla Z^{*}$ slowly. In FastSME as shown in dark green in Figure 1B, the foreground local consistency adjustment and foreground to background manifold propagation happens simultaneously resulting in a steeper drop in $\nabla Z^{*}$. Additionally, as a consequence of simultaneous corrections of index map for both foreground and background, the cost minima $\nabla Z^{*}$ reachable is even lower than that of SME with significantly fewer iterations.

\subsection{Multi-scale pyramidal index map optimization}

For very large images, it is more efficient to learn to estimate the index map in lower resolution at early stages of the optimization and use the partially optimized manifold to initialize for the full resolution optimization. As the low resolution optimization result is already close to the final desired local minima, it takes fewer iterations to converge on the full resolution index map. By lowering the resolution by 0.5 , we could reduce the number of pixel locations to optimize by $1 / 4$ th. We implemented the pyramid scheme as a power of $2^{9}$ implying if the image is more than of size $512 X 512$, it will have 2 stage iteration process where first stage would be $50 \%$ of the full resolution. For images of size more than $1024 \times 1024$ it would have 3 stage of sequential $25 \%, 50 \%$ and $100 \%$ resolution optimization and so forth. Working initially at lower resolution helps getting a smoother manifold as it initially reconstructs a flatter manifold and later corrects the local variations. Additionally, in lower resolution the local smoothing window effectively becomes bigger compared to when performed on full resolution and thereby helps propagate the foreground manifold information faster to the reachable background pixels. Figure 2 presents a visual illustrations of the differences in index map created by FastSME and SME on 'EPENDYMAL CELLS' dataset (2 A) using the class map in Figure 2 B. In Figure 2 C, we can see that the FastSME index map on the left appears smoother and more similar to the actual biological structure of the 'EPENDYMAL CELL' apical tissue layer. Whereas in SME index map on the right (Figure $2 \mathrm{D}$ ), there are some examples of bright yellow points within blue areas representing some artifacts extracted from depth of the cell rather than being on the apical layer. This multiscale pyramid index map optimization strategy significantly reduces the number of computation in each iteration during optimization and provides robust performance across a large variety of biological structures as shown in the result section 6 .

In the pilot SME framework, the class map was created using kmeans on the frequency domain representation of z-profile of each pixel. Performing kmeans clustering on very large dataset can consume huge amount of resources in terms of memory and time, which is now parallelized in FastSME. Parallelized kmeans clustering for smaller images does not make much difference in terms of speed but for large dataset it is significant.

In figure 3, the computations time of SME and FastSME has been tested on 10 dataset and presented ordered by the increasing number of pixels of the index map Width $\times$ Height . The computation time recorded is the total execution time of SME and FastSME. Execution time includes and can be dependent on loading time of the data, finding multichannel projections using the reference index map of the selected channel, the quality of the initialization based on MIP. But the majority of the computation time is the taken by the iterative energy optimization scheme. As illustrated in figure 3, we achieved an average speed gain of $7 X$ in FastSME compared to that of SME. The gain in speed with FastSME is higher in larger dataset keeping the quality of the composite image and index map intact as described in the following results section.

\section{Results and Evaluation}

\subsection{Dataset}

In this work, we primarily test all the dataset reported in the SME paper [15]. We did not use the 'EPENDYMAL CELL-TILE SCAN' image due to it being a larger mosaic of the reported 'EPENDYMAL CELLS' dataset, rather introduced a new dataset of golgi bodies acquired in confocal imaging 'GOLGI'. The quantitative comparison was performed on the 'SYNTHETIC TISSUE' data. The method for creating this particular dataset is described in [15]. 


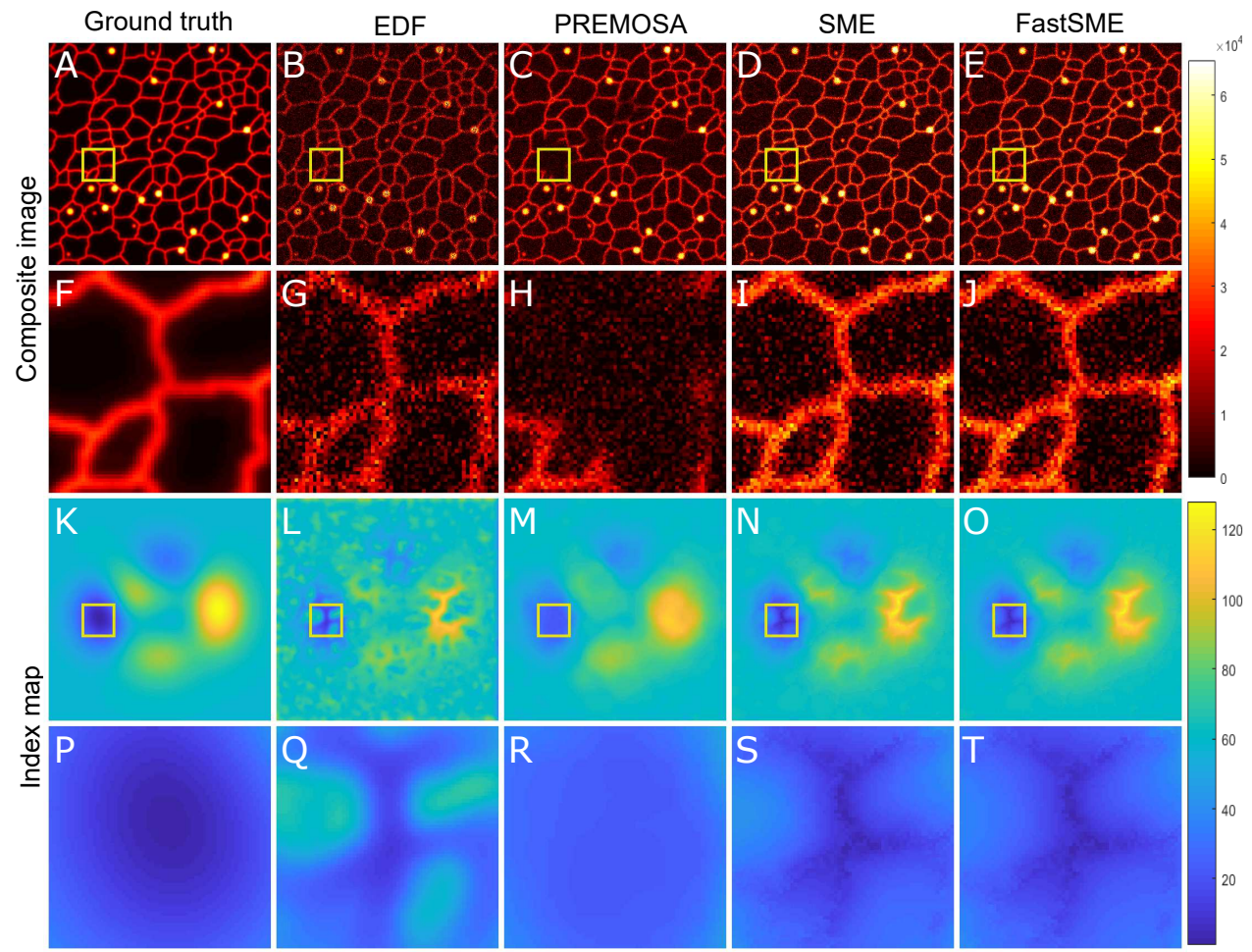

Figure 4. Visualization of recovered composite image and index map on synthetic data. The 2nd and 4th rows are the zoomed view of yellow square in 1st and 3rd rows to demonstrate clearly the performance the various methods. Both EDF and Premosa fail to recover parts of the synthetic data and the reason for that is the erroneous index maps they create. EDF recovers neighboring foreground and background pixels from different depths of the image stack. (compare P,Q) and Premosa creates a over-smoothed index map not following the data (compare P,R). FastSME can follow the actual manifold more accurately (compare P,T) and hence recovers the most authentic composite image from 3D stacks.

\subsection{Evaluation metrics}

We evaluate the performance of our proposed method with multiple metrics scoring the quality of the reconstructed composite image w.r.t. the reference image data - normalized mutual information and signal-to-noise ratio; the fidelity of the recovered index map with root mean square error w.r.t. the reference index map used to create the synthetic data; and finally on the time taken by each of the methods.

- Firstly we compare the normalized mutual information (nMI) content of the recovered composite image $I_{X}$ to study the co-relation with the reference ground truth image $I_{R}$, before corrupting the data with PSF and noise, mimicking a real microscopy image.

$$
\mathrm{nMI}=\sum_{x} \sum_{y} p\left(I_{X}, I_{R}\right) \log \left(\frac{p\left(I_{X}, I_{R}\right)}{p\left(I_{X}\right) p\left(I_{R}\right)}\right),
$$

where $p\left(I_{X}\right)$ and $p\left(I_{R}\right)$ are the marginal probability density functions of the recovered composite image $I_{X}$ and reference image $I_{R}$ respectively. The physical significance of this metric is it enables to evaluate how much of the original information content of the reference $2 \mathrm{D}$ image is retained in the recovered composite image from the $3 \mathrm{D}$ volume.

- The second metric we use is the Signal to Noise Ratio (SNR) in decibel scale -

$$
\mathrm{SNR}=20 \log \left[\frac{\sigma^{2}\left(I_{R}\right)}{\sigma^{2}\left(I_{R}-I_{X}\right)}\right]
$$

where $S N R$, is the ratio of variance of the reconstructed composite image $I_{R}$ and the variance of the noise which is the difference between the reconstructed composite image $I_{R}$ and the ground truth image $I_{X}$. The metric gives an indication of how much of the distortions due to PSF and sensor noise is covered from the image volume.

- To estimate the precision of the reconstructed index map $Z_{R}$, it is compared in terms of root mean squared error (RSME) with the synthetic index map $Z_{X}$ (which is used to generate the 'SYNTHETIC TISSUE' dataset). 

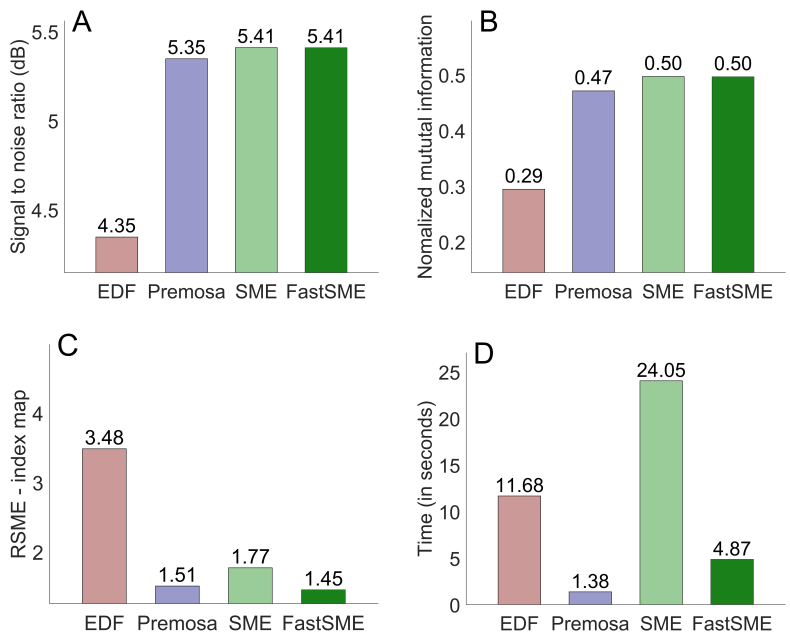

Figure 5. Performance evaluation on synthetic data with multiple metrics. Premosa [4], SME [15] and FastSME are comparable on SNR(A) and NMI(B), and infact speed wise FastSME is slower than Premosa (D). However, FastSME outperforms all methods on the accuracy of recovered manifold (C-RMSE- index map) which has important implications for the quality of composite image as shown in Fig. 4.

$$
\operatorname{RSME}=\sqrt{\frac{\sum_{x, y}\left(Z_{X}(x, y)-Z_{R}(x, y)\right)^{2}}{W \times H}}
$$

where $W$ and $H$ are the width and height of the image respectively.

- Finally, the speed is compared among all these methods based on time required for execution keeping all the parameter fixed to the default values.

The performance of EDF, Premosa, the pilot SME methods and our proposed method FastSME w.r.t. the metrics SNR, nMI, RMSE and speed are presented in Fig. 5 . We chose these algorithms due to their easy accessibility in the microscopy image analysis community as ImageJ plugins that make them popular with bioimage analysts. We also compared the results of FastSME with maximum intensity projection (MIP) which is the most commonly ( $90 \%$ of the time) used tool by biologists, as reported in a survey in [15]. SME and FastSME performed slightly better than Premosa w.r.t. metrics SNR and nMI 4 $\mathrm{B}, \mathrm{A}$ indicating the better quality of the composite image. When evaluated on the accuracy of the index map recovery in terms of RSME w.r.t. the ground truth index map, as illustrated in Fig 5 C, FastSME generates the most precise index map with the lowest error among all. Premosa, which is the fastest among all the reported methods, also performed very well in terms of RSME of index map however missed some high curvature points as shown in Figure $4 \mathrm{C}$ and $\mathrm{H}$.

\subsection{Results on diverse microscopy data}

The algorithm is applied on multiple diverse datasets from different microscopy modalities 6 A-T. For details regarding the preparation of the datasets the interested reader is referred to the pilot SME paper [15]. A, B illustrating all-in-focus reconstruction of neuronal branches with FastSME compared to MIP on 'NEURON1'. C, D represents MIP and SME projections on 'TUBULIN' widefield image where MIP failed to reconstruct the detailed actin network whereas FastSME successfully depicted them. E, F represents MIP and SME projections on 'MEMBRANE1' confocal image showing contrast enhanced denoised projection by FastSME. G, H represents MIP and SME projections on 'NEURON2' confocal image showing lower background noise accumulation by FastSME. I, J represents MIP and SME projections on 'GOLGI' confocal image illustrating cleaner projection by FastSME. K, L represents MIP and SME projections on 'SYNTHETIC TISSUE' confocal image illustrating higher denoising by FastSME. M, N represents composite images from 2-channel 'DENDRITE' dataset with dendrites (red channel) as reference for index map, illustrating the colocalized buttons around the dendrites and lower background noise with FastSME than with MIP. O, P represents MIP and SME projections on 'NUCLEI' 3 channel confocal image with membrane in red as reference, showing that FastSME could locally reconstruct cleaner and across channel coherent projection with apical layer than by MIP. Q, R represents MIP and SME projections on 3 channel 'CANCER CELLS' confocal image with nucleus in blue as reference, showing accurate localized membrane projection around nucleus center along $\mathrm{z}$ by FastSME. $\mathrm{S}, \mathrm{T}$ represents MIP and SME projections on 3 channel 'EPENDYMAL CELL' confocal image with membrane in blue as reference, illustrating that FastSME projection could reconstruct localized projection of centrioles and pro-centrioles with apical layer without accumulating unwanted blood vessels from the depth of the volume.

Overall, FastSME is able to generate very precise index map and well contrasted locally coherent accurate composite images with relatively high speed. Premosa is very consistent even in very noisy image in terms of accuracy and higher speed, however is limited to confocal imaging only, whereas SME and FastSME can work with both confocal and widefield images. FastSME can be used for multi channel projection applying the index map from user defined reference channel to acquire data along the manifold of interest from other channels. In this way, we can extract $2 \mathrm{~d}$ projection of a $3 \mathrm{D}$ stack maintaining within and between channel local spatial consistency w.r.t the index map reconstructing closer approximation of the real 3D manifold of the biological object of interest. 


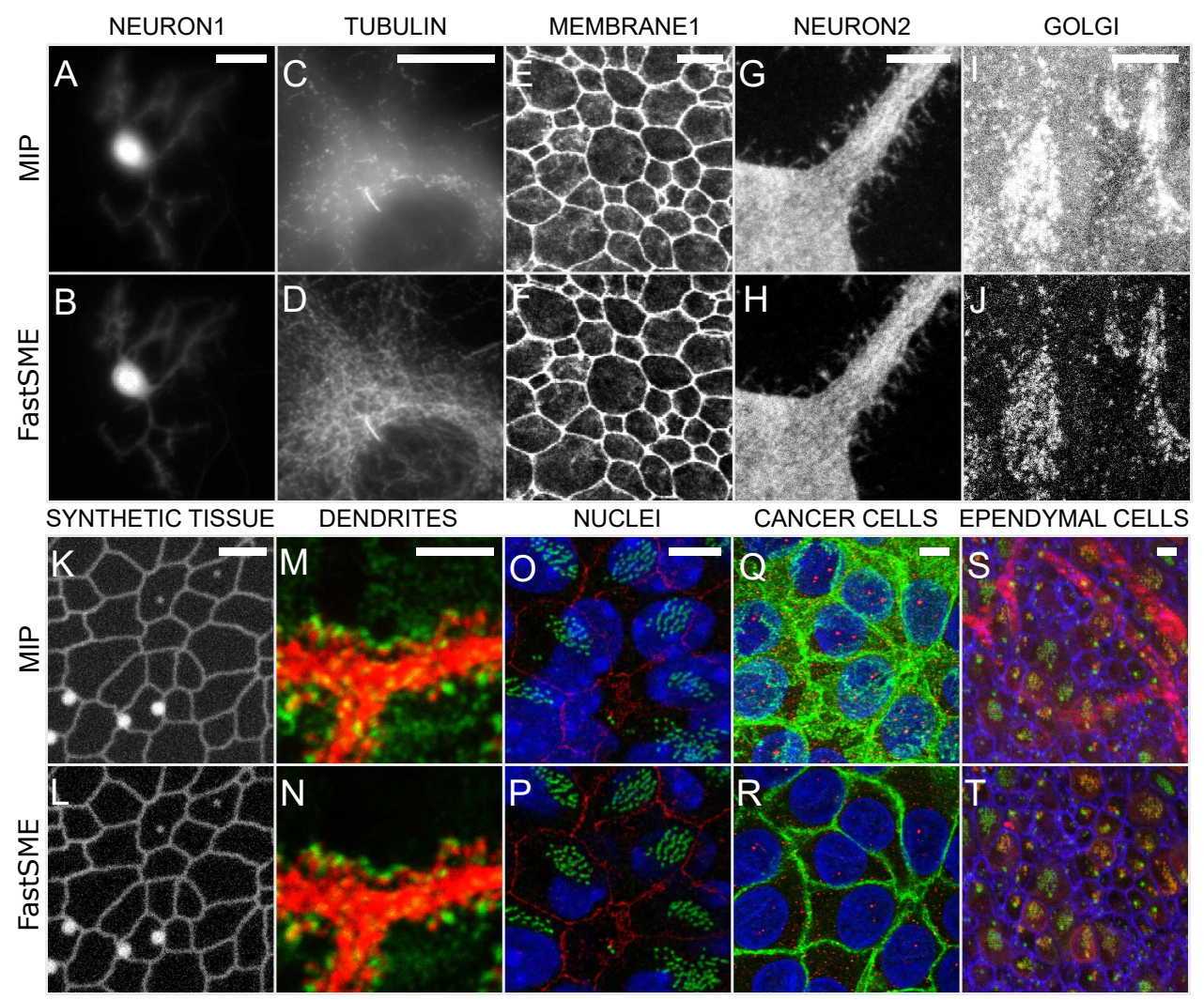

Figure 6. Comparison among composite images created by MIP and FastSME method on varied single (in grayscale) or multichannel datasets acquired by widefield (NEURON1 and TUBULIN) and confocal (rest except SYNTHETIC TISSUE) microscopes. Scale bar is 5 micron. In all the cases presented here, FastSME generates denoised, contrast enhanced, and spatially coherent representation of the morphology of the biological specimen when compared with MIP (most commonly used pixel wise projection method).

FastSME is currently implemented on MATLAB and all reported results are obtained on MATLAB $2017 \mathrm{~b}$ on Intel core i7-7700 HQ CPU 2.81GHz 32GB ram ZBook laptop. The codes are made publicly available at https://github.com/Shihav/FastSME and all the dataset used for this work is being made publicly available at https://data.mendeley.com/datasets/bn7zbzc3gg/1. In the near future, we plan to make available a Fiji plugin for FastSME following the suggestion of the community for easier accessibility and adoption of the method to increase the potential impact of FastSME. We will include features to make it easier for FastSME users to - 1) automatically update via ImageJ/Fiji update mechanism 2) deploy command-line version of the library for use in high-throughput and multi-threading (several instances of FastSME running in parallel) in cluster environments.

\section{Conclusion}

In this paper, we propose FastSME, an improved version of the SME method that allows faster and more acurate $2 \mathrm{D}$ representation of data lying on a smooth manifold in a $3 \mathrm{D}$ volume generated by common microscopy modalities. The improvements are achieved mainly through adoption of a better adapted cost function design and sophisticated optimization strategy with stochastic gradient descend momentum optimization embedded in a simulated annealing schema coupled with a multi-scale pyramidal index map optimization. We reported on average 7X gain in speed compared to baseline SME and improved index map and composite image precision compared to state-of-the-art methods in terms of multiple metrics. FastSME can be reliably used as 2D projection method of specimen morphology lying on a smooth 2.5D manifold in 3D space. This has wide ranging applications in bioimage analysis as demonstrated by the many real data examples presented in the paper, for example, in colocalization study across multiple channels.

\section{References}

[1] F. Aguet, D. Van De Ville, and M. Unser. Model-based 2.5-D deconvolution for extended depth of field in brightfield microscopy. IEEE Transactions on Image Processing, 17(7):1144-1153, July 2008. 1, 2

[2] A. Al Jord, A. Shihavuddin, R. S. daout, M. Faucourt, A. Genovesio, A. Karaiskou, J. Sobczak-Thepot, N. Spassky, and 
A. Meunier. Calibrated mitotic oscillator drives motile ciliogenesis. Science, 358(6364):803-806, 2017. 2

[3] X. Bai, Y. Zhang, F. Zhou, and B. Xue. Quadtree-based multi-focus image fusion using a weighted focus-measure. Information Fusion, 22:105-118, 2015. 1

[4] C. Blasse, S. Saalfeld, R. Etournay, A. Sagner, S. Eaton, and E. W. Myers. Premosa: extracting 2d surfaces from $3 \mathrm{~d}$ microscopy mosaics. Bioinformatics, 33(16):2563-2569, 2017. 2,7

[5] M. Boshtayeva, D. Hafner, and J. Weickert. A focus fusion framework with anisotropic depth map smoothing. Pattern Recognition, 2014. 1

[6] P. S. Bradley and U. M. Fayyad. Refining initial points for kmeans clustering. In ICML, volume 98, pages 91-99. Citeseer, 1998. 3

[7] A. E. Carpenter, T. R. Jones, M. R. Lamprecht, C. Clarke, I. H. Kang, O. Friman, D. A. Guertin, J. H. Chang, R. A. Lindquist, J. Moffat, et al. Cellprofiler: image analysis software for identifying and quantifying cell phenotypes. Genome biology, 7(10):R100, 2006. 2

[8] R. Etournay, M. Merkel, M. Popović, H. Brandl, N. A. Dye, B. Aigouy, G. Salbreux, S. Eaton, and F. Jülicher. Tissueminer: a multiscale analysis toolkit to quantify how cellular processes create tissue dynamics. Elife, 5, 2016. 2

[9] P. Foerster, M. Daclin, S. Asm, M. Faucourt, A. Boletta, A. Genovesio, and N. Spassky. mtorcl signaling and primary cilia are required for brain ventricle morphogenesis. Development, 144(2):201-210, 2017. 2

[10] D. P. Kingma and J. Ba. Adam: A method for stochastic optimization. arXiv preprint arXiv:1412.6980, 2014. 4

[11] N. Marturi, S. Dembélé, and N. Piat. Depth and shape estimation from focus in scanning electron microscope for micromanipulation. In International Conference on Control, Automation, Robotics \& Embedded Systems, CARE'13., pages 1-6, 2013. 1

[12] N. Qian. On the momentum term in gradient descent learning algorithms. Neural networks, 12(1):145-151, 1999. 2,4

[13] C. A. Schneider, W. S. Rasband, and K. W. Eliceiri. Nih image to imagej: 25 years of image analysis. Nature methods, 9(7):671-675, 2012. 2

[14] J. Selinummi, P. Ruusuvuori, I. Podolsky, A. Ozinsky, E. Gold, O. Yli-Harja, A. Aderem, and I. Shmulevich. Bright field microscopy as an alternative to whole cell fluorescence in automated analysis of macrophage images. PloS one, 4(10): $\mathrm{e} 7497,2009.1$

[15] A. Shihavuddin, S. Basu, E. Rexhepaj, F. Delestro, N. Menezes, S. M. Sigoillot, E. Del Nery, F. Selimi, N. Spassky, and A. Genovesio. Smooth 2d manifold extraction from $3 \mathrm{~d}$ image stack. Nature Communications, 8:15554, 2017. 1, 2, 3, 4, 5, 7

[16] S. G. Stanciu, F. J. Ávila, R. Hristu, and J. M. Bueno. A study on image quality in polarization-resolved second harmonic generation microscopy. Scientific reports, 7(1):15476, 2017. 2

[17] S. G. Stanciu, M. Dragulinescu, and G. A. Stanciu. Summodified-laplacian fusion methods experimented on im- age stacks of photonic quantum ring laser devices collected by confocal scanning laser microscopy. University" Politehnica" of Bucharest Scientific Bulletin, Series A: Applied Mathematics and Physics, 73(2):139-146, 2011. 1

[18] T. Tieleman and G. Hinton. Lecture 6.5-rmsprop: Divide the gradient by a running average of its recent magnitude. COURSERA: Neural networks for machine learning, 4(2):26-31, 2012. 4

[19] M. Weigert, U. Schmidt, T. Boothe, M. Andreas, A. Dibrov, A. Jain, B. Wilhelm, D. Schmidt, C. Broaddus, S. Culley, et al. Content-aware image restoration: Pushing the limits of fluorescence microscopy. bioRxiv, page 236463, 2017. 2

[20] M. D. Zeiler. Adadelta: an adaptive learning rate method. arXiv preprint arXiv:1212.5701, 2012. 4 